\title{
Carcinoma mucoepidermóide de brônquio: uma rara causa de pneumonia recorrente em uma criança
}

\author{
Mucoepidermoid carcinoma of bronchus: a rare cause of recurrent pneumonia in a child \\ Daniel Cury Ogata'; Sérgio Ossamu loshii'; Ricardo Reis Blum³ ${ }^{3}$ Paulo Henrique Dondoni4; \\ Sérgio Kenji Akamine ${ }^{5}$; Fernando Schuster Battaglin ${ }^{6}$; Mara Albonei Dudeque Pianovski ${ }^{7}$
}

\begin{tabular}{l}
\multicolumn{1}{c|}{ Unitermos } \\
Carcinoma mucoepidermóide \\
Pneumonia recorrente \\
Broncoscopia
\end{tabular}

resumo

Os autores relatam o caso de um paciente de 13 anos com tosse crônica e pneumonia recorrente, que revelou, na broncoscopia, massa vegetante obstruindo brônquio segmentar superior direito. Foi realizada biópsia, a qual revelou carcinoma mucoepidermóide de baixo grau. O paciente foi submetido posteriormente a bilobectomia. Atualmente encontra-se bem, em seguimento de 14 meses em nosso serviço.

\section{Introdução}

O carcinoma mucoepidermóide broncogênico é uma rara neoplasia, representando menos de $0,5 \%$ dos tumores malignos broncopulmonares primitivos ${ }^{(5)}$. São exclusivamente endobrônquicos e vegetantes ${ }^{(3)}$. Histologicamente caracterizam-se por conter variável proporção de células escamosas e secretoras de muco, além das células intermediárias ${ }^{(4)}$. Os sintomas relatados são, na maioria das vezes, decorrentes da obstrução brônquica, como atelectasia persistente ou pneumonia recorrente ${ }^{(3)}$.

\section{abstract}

The authors report a case of patient of 13 years of age that presented chronic cough and recurrent pneumonia, whose bronchoscopy showed a polipoid mass obstructing upper right segmental bronchus. Biopsy was performed and revealed low-grade mucoepidermoid carcinoma. Subsequently, the patient was submitted to a bilobectomy. Nowadays, the patient has been met well in follow-up of 14 months in our department. key words

Mucoepidermoid carcinoma

Recurrent pneumonia

Bronchoscopy

\section{Relato de caso}

Paciente do sexo masculino, 13 anos, procurou atendimento médico devido à queixa de tosse seca há aproximadamente quatro semanas e cinco episódios de pneumonia nos últimos seis meses. Não apresentava alterações no exame físico. Na ocasião foi realizada

\footnotetext{
1. Médico patologista do Hospital Erasto Gaertner.

2. Chefe do Departamento de Anatomia Patológica do Hospital Erasto Caertner.

3. Médico-residente em Oncologia Pediátrica do Hospital Erasto Gaertner.

4. Médico-residente em Cirurgia Oncológica do Hospital Erasto Gaertner.

5. Médico radiologista do Hospital Erasto Gaertner.

6. Acadêmico de Medicina do Hospital Erasto Gaertner.

7. Chefe do Departamento de Oncologia Pediátrica do Hospital Erasto Caertner.
} 
radiografia de tórax, que mostrou área sólida e consolidada em lobo superior direito (Figura 1). O paciente foi então encaminhado para o Hospital Erasto Gaertner para investigação. Nesse hospital foi realizada tomografia axial computadorizada (TAC) de tórax sem contraste. O referido exame revelou massa de contornos irregulares, sem plano de clivagem definido com estruturas mediastinais (Figura 2). Com base nesses resultados, optou-se por fazer uma broncoscopia, na qual foi visualizada massa vegetante e bocelada com aproximadamente $1 \mathrm{~cm}$ de diâmetro, preenchendo brônquio segmentar superior direito, realizando-se, então, biópsia da lesão. $\mathrm{O}$ diagnóstico histopatológico foi carcinoma mucoepidermóide de baixo grau associado a pneumonia lipóide. Posteriormente foi proposto como tratamento a bilobectomia.

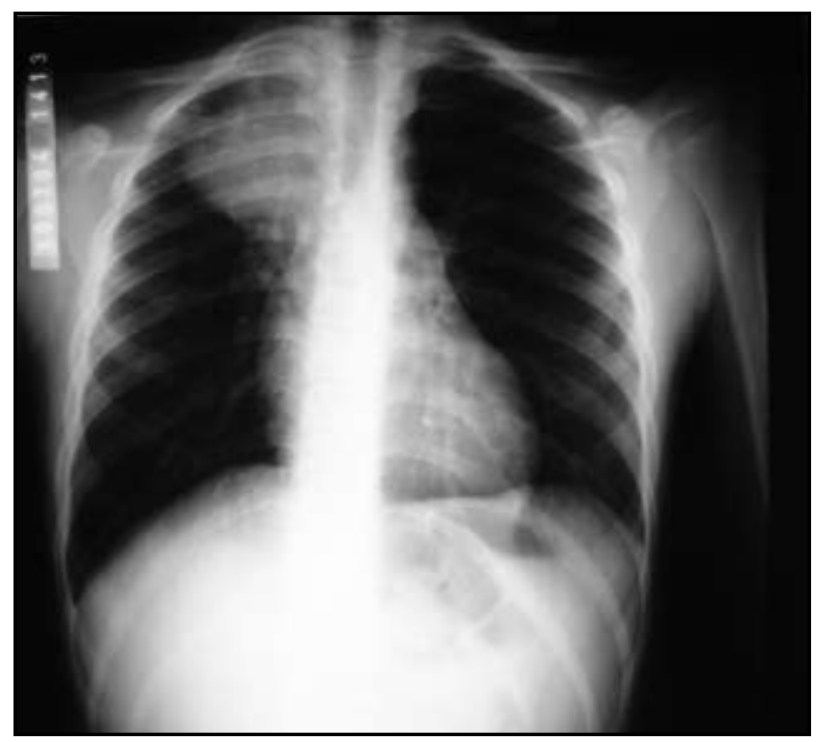

Figura 1 - Radiografia de tórax mostrando opacidade de limites parcialmente bem definidos de lobo superior de pulmão direito em contato íntimo com mediastino

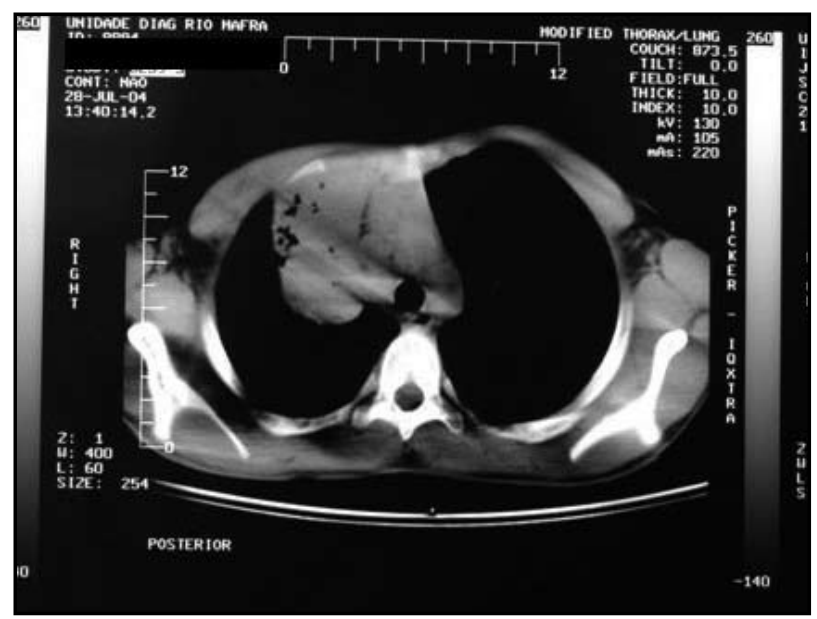

Figura 2 - Tomografia axial computadorizada de tórax sem contraste, revelando massa peri-hilar localizada em segmento anterior de lobo superior direito, sendo que distalmente observa-se área de consolidação com focos de densidade de gordura de permeio
O exame macroscópico mostrou tumor vegetante, pardacento e bocelado com $1,3 \mathrm{~cm} \times 1 \mathrm{~cm}$, com localização endobrônquica, sem invasão de parênquima. Microscopicamente a neoplasia era constituída por ilhas de células escamosas com baixo pleomorfismo, entremeada por moderada quantidade de células produtoras de muco, além de microcalcificações difusas (Figuras 3A, 3B, 3C e 3D). Não havia necrose e o índice mitótico era menos de um em 10 campos de grande aumento. O diagnóstico prévio foi então confirmado.

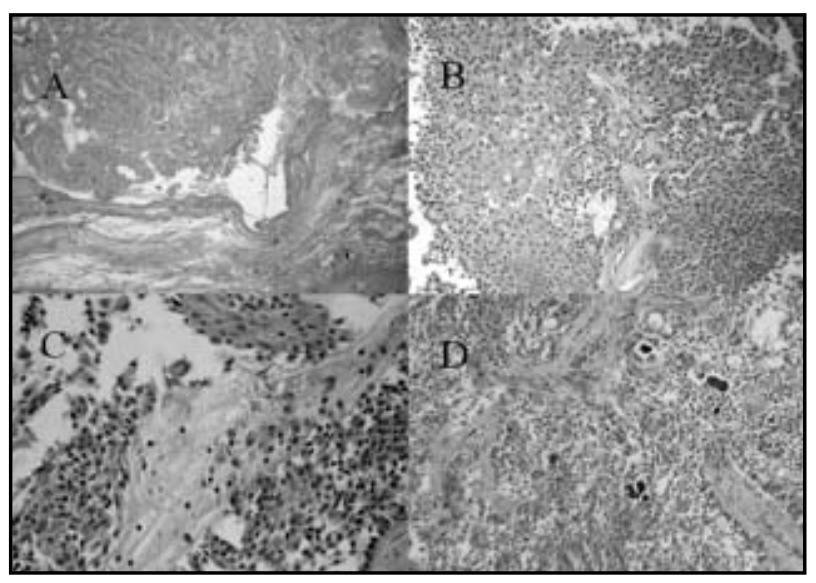

Figura 3 - A microscopia do tumor revela massa endoluminal (A) constituída por blocos de células escamosas com atipias discretas, (B) eventualmente sendo observados grupos celulares mucossecretores (C). Calcificações distróficas difusas foram evidenciadas (D) (coloração de hematoxilina-eosina, a foto $A$ com aumento de $40 x$, as fotos $B$ e $D$ de $100 x$ e a foto $C, 400 x$ )

Após a cirurgia optou-se apenas por seguir o paciente, não sendo realizado nenhum tratamento adicional. 0 paciente encontra-se bem em seguimento de 14 meses em nosso serviço.

\section{Discussão}

Os tumores mucoepidermóides de pulmão foram descritos inicialmente por Smetana, em $1952^{(8)}$, e desde então aproximadamente 60 casos foram relatados na literatura ${ }^{(9)}$. A idade dos pacientes variava de 3 meses a 78 anos, contudo aproximadamente metade apresentava menos de 30 anos $^{(6)}$.

Estas neoplasias apresentam-se na forma de massa polipóide endoluminal, localizando-se freqüentemente em brônquios segmentares ou lobares e sendo raramente observadas na traquéia ou nos brônquios principais ${ }^{(4)}$. Os sintomas são principalmente referentes à obstrução de vias aéreas e incluem pneumonia recorrente, atelectasia persistente, tosse crônica e, menos freqüentemente, hemoptise ${ }^{(3,6)}$. Pacientes assintomáticos variavam de $9 \%$ a $28 \%{ }^{(1)}$. A média de duração dos sintomas antes do diagnóstico era de 1,5 ano ${ }^{(9)}$. 
A alteração radiográfica mais freqüente é o nódulo solitário (71\%), seguido de consolidação pneumônica $(28 \%)^{(4)}$. Apenas um caso relatado na literatura apresentou radiografia de tórax dentro dos limites da normalidade. A tomografia computadorizada (TC) mostra um tumor de limites lobulados ou ovalados, homogêneo, podendo conter calcificação e com captação discreta de contraste ${ }^{(6)}$. Linfadenomegalia hilar ou mediastinal é raramente observada ${ }^{(6)}$. Achados associados, como dilatação brônquica, impactação de muco, pneumonia pós-obstrutiva ou atelectasia segmentar, são sugestivos de massa endobrônquica ${ }^{(4)}$.

Macroscopicamente, este tumor apresenta-se como massa endobrônquica polipóide, bem circunscrita e, devido à localização central, é facilmente visualizada na broncoscopia ${ }^{(9)}$. Na microscopia esta neoplasia tem aparência sólido-cística, com presença de elementos celulares escamosos, células produtoras de muco e células intermediárias ou basais ${ }^{(9)}$. Focos de calcificação, presentes neste relato, ou ossificação têm sido relatados ${ }^{(4)}$. A incidência de calcificação nos carcinomas mucoepidermóides de pulmão é muito maior quando em comparação com as formas mais comuns de carcinomas pulmonares ${ }^{(4)}$. Infiltrado linfóide exuberante e grau variável de células gigantes multinucleadas, além de alterações de células claras, têm sido descritos, podendo dificultar o diagnóstico(7). Os tumores mucoepidermóides são morfologicamente divididos em baixo e alto grau de malignidade. As mitoses, o pleomorfismo nuclear e a necrose são geralmente mínimos ou ausentes nos carcinomas mucoepidermóides de baixo grau. Nos tumores de alto grau, as mitoses são abundantes, com média de quatro em 10 campos de grande aumento. O pleomorfismo nuclear é mais evidente e a necrose celular está presente ${ }^{(4)}$.

Em uma considerável proporção dos carcinomas mucoepidermóides, a translocação $t(11 ; 19)$ é a única anomalia genômica presente, indicando uma alteração citogenética primária. A correlação do curso clínico e a presença de aberrações citogenéticas adicionais podem indicar pior prognóstico(2).

O tratamento consiste em remoção cirúrgica cuidadosa do tumor, além de linfonodos regionais e tecido pulmonar normal como margem. Quando tecnicamente possível, a ressecção "em manga" do brônquio envolvido é recomendável. No entanto, na maioria dos casos, a localização da lesão requer lobectomia ou pneumectomia. A excisão broncoscópica de tumores mucoepidermóides de baixo grau tem sido relatada na literatura, mas não é recomendada devido ao risco de recorrência local ${ }^{(3)}$. Os carcinomas mucoepidermóides não são sensíveis a radioterapia ou quimioterapia ${ }^{(9)}$.

Os tumores mucoepidermóides de baixo grau, quando completamente ressecados, têm excelente prognóstico, com baixa chance de recorrências ou metástases ${ }^{(4)}$. As lesões de alto grau têm melhor prognóstico que os carcinomas broncogênicos comuns ${ }^{(4)}$. Em um estudo envolvendo 13 pacientes com carcinomas mucoepidermóides de alto grau, apenas três faleceram devido à doença (dois deles com metástases em linfonodos regionais) ${ }^{(10)}$. Resumindo, esta neoplasia é insidiosa e de crescimento lento, cujos sintomas são referentes à obstrução brônquica. Contudo, algumas vezes são inespecíficos, dificultando o raciocínio semiológico e retardando o diagnóstico, que geralmente é feito tardiamente.

\section{Referências}

I. COLBY,T.V.; KOSS, M. N.;TRAVIS, W. D.Tumors of salivary gland type. In: Tumors of the lower respiratory tract: AFIP atlas of tumor pathology. $3^{\text {rd }}$ series, Washington. DC:American Registry of Pathology, 1995. v. I3, p. 65-9.

2. EL-NAGGAR, A. K. et al. A mucoepidermoid carcinoma of minor salivary gland with $\mathrm{t}(1$ I; 19) (q21;p I3, I) as the only karyotypic abnormality. Cancer Genet Cytogenet, v. 87, p. 29-33, 1996.

3. FAUROUX, B. et al. Carcinoid and mucoepidermoid bronchial tumors in children. Eur J Pediatr, v. I64, p. 748-52, 2005.

4. KIM,T.S. et al. Mucoepidermoid carcinoma of the tracheobronchial tree: radiographic and CT findings in 12 patients. Radiol, v. 212, p. 643-8, 1999
5. LEBEAU, B.; GENOT, C. Carcinome bronchique mucoépidermoïde chez um fumeur de cannabis age de 22 ans. Presse Med, v. 34, p. I229-32, 2005.

6. PANDYA, H.; MATTHEWS, S. Mucoepidermoid carcinoma in a patient with congenital agenesis of the left upper lobe. The British Journal of Radiology, v. 76, p. 339-42, 2003.

7. SHILO, K. et al. Pulmonary mucoepidermoid carcinoma with prominent tumor-associated lymphoid proliferation. Am J Surg Pathol, v. 29, n. 3, p. 407-I I, 2005.

8. SMETANA, G. et al. Bronchogenic carcinoma: an analysis of 100 autopsy cases. Milit Surg, v. I I I, p. 335-5 I, 1952. 
9.VOGELBERG, C. et al. Mucoepidermoid carcinoma as an unusual cause for recurrent respiratory infections in a child. J Pediatr Hematol Oncol, v. 27, n. 3, p. 162-5, 2005.
10. YOUSEN, S. A.; HOCHHOLZER, L. Mucoepidermoid tumors of lung. Cancer, v. 60, p. 1346-52, 1987. 